Faculdade

de Ciências Econômicas UFRGS
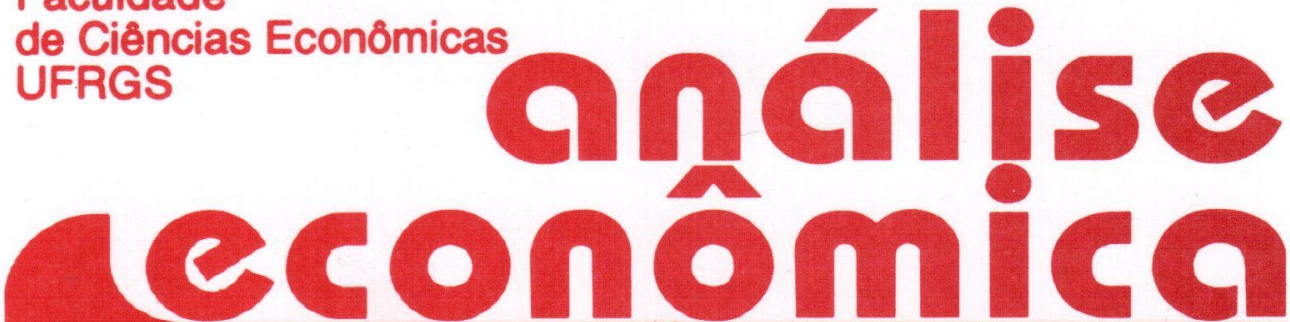

- EQUILIBRIO, PROGRESSO TÉCNICO E DESIGUALDADES REGIONAIS

Carlos Roberto Azzoni

- DESENVOLVIMENTO POLARIZADO E DESEQUILÍBRIOS REGIONAIS Nali de Jesus de Souza

- OS NOVOS CLÁSSICOS E O MÉTODO Carlos Magno Lopes

- ECONOMLAS DE MERCADO E DEMANDA EFETIVA Gilberto Tadeu Lima

- MEASURES OF CAPACITY UTILIZATION Marcelo S. Portugal

- ASPECTOS DO CONTROLE EM UM MODELO DINÂMICO

Marat Rafikow

Pedro Augusto P. Borges

- A FIRMA EM UM AMBIENTE INFLACIONÁRIO

Carmen A.do V.C. Feijó

- CUSTOS E BENEFICIOS DA INTEGRAÇĀO REGIONAL Marco Antônio Montoya

- A REESTRUTURAÇÃO DA ECONOMIA MUNDIAL Hoyêdo Nunes Lins

- O MERCADO COMO PROCESSO: A ABORDAGEM AUSTRÍACA

Fernando Caputo Zanella

- GARY BECKER: PRÊMIO NOBEL DE ECONOMIA DE 1992

Giácomo Babinotto Neto

LIVROS RECEBIDOS

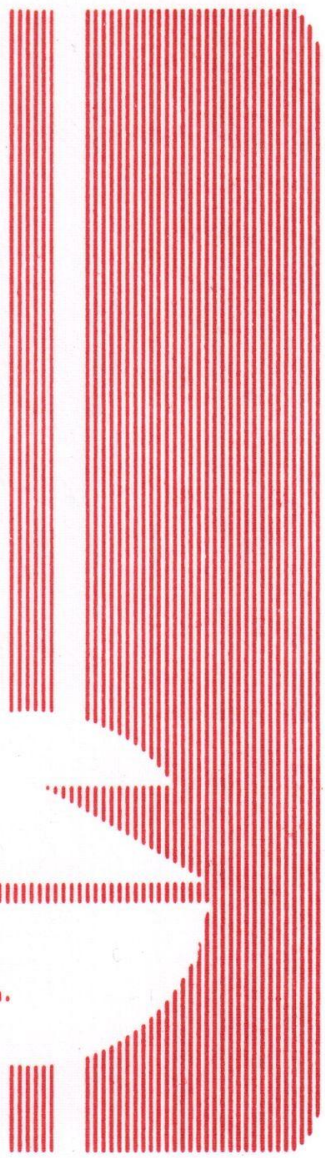


UNIVERSIDADE FEDERAL DO RIO GRANDE DO SUL Reitor: Prof. Hélgio Henrique Casses Trindade

FACULDADE DE CIENCIAS ECONOMICAS

Diretor: Prof. Pedro Cézar Dutra Fonseça

CENTRO DE ESTUDOS E PESQUISAS ECONOMICAS

Diretor: Prof. Roberto Pires Pacheco

DEPARTAMENTO DE CIENCIAS ECONÓMICAS

Chefe: Prof. Fernando Ferrari Filho

CURSO DE POS-GRADUAÇAOO EM ECONOMIA

Coordenador: Prof. Joăo Rogério Sanson

CURSO DE POS-GRADUAÇÂO EM ECONOMIA RURAL

Coordenador: Prof. Juvir Luiz Mattuella

CONSELHO EDITORIAL: Achyles Barcelos da Costa, Aray Miguel Feldens, Atos Freitas Grawunder, Carlos Augusto Crusius, Ernani Hickmann, Fernando Ferrari Filho, Joāo Rogério Sanson, Juvir Luiz Mattuella, Marcelo Savino Portunal, Maria Imilda da Costa e Silva, Nali de Jesus de Souza, Nuno Renan Lopes de Figueiredo Pinto, Otília Beatriz Kroeff Carrion, Otto Guilherme Konzen, Paulo Alexandre Sphor, Pedro Cezar Dutra Fonseca, Reinaldo I gnacio Adams, Roberto Camps Moraes, Valter José Stülp, Yeda Rorato Crusius, David Garlow (Wharton Econometrics Forecasts Association, E.U.A.), Edgar Augusto Lanzer (UFSC), Eleutério F. S. Prado (USP), Fernando Holanda Barbosa (FGV/RJ), Gustavo Franco (PUC/RJ), Joaquim Pinto de Andrade (UnB), Juan H. Moldau (USP), Werner Baer (Univ. de lllinois, E.U.A.).

COMISSĀO EDITORIAL: Atos Freitas Grawunder, Pedro Cezar Dutra Fonseca, Reinaldo Ignacio Adams e Roberto Camps Moraes.

EDITOR: Prof. Nali de Jesus de Souza

SECRETARIA: Maria Ivone de Mello (normalizaçāo), Vanete Ricacheski (revisão de textos).

FUNDADOR: Prof. Antônio Carlos Santos Rosa

Os materiais publicados na revisța Análise Econômica sāo da exclusiva responsabilidade dos autores. E permitida a reprodıçāo total ou parcial dos trabalhos, desde que seja citada a fonte.

Aceita-se permuta com revistas congêneres. Aceitam-se, também, livros para divulgaçāo, elaboraçāo de resenhas ou recensōes.

Toda correspondência, material para publicação (vide normas na terceira capa), assinaturas e permutas devem ser dirigidos ao seguinte destinatário:

\author{
PROF. ROBERTO CAMPS MORAES \\ Revista Análise Económica \\ Av. Joāo Pessoa, 52 \\ CEP 90040-000 - PORTO ALEGRE (RS), BRASIL \\ Telefones: (051) 228-1633, ramal 3440
}

Fax: (051) 225-1067 


\section{DISSERTAÇÕES DE MESTRADO DEFENDIDAS NOS CURSOS DE PÓS-GRA- DUAÇÃO EM ECONOMIA E EM ECONO- MIA RURAL DA UFRGS, EM 1992}

\section{A) ECONOMIA}

BELL.O, Teresinha da Silva. Teoria Econômica e Relaçöes Internacionais: da Pilhagem àMundialização do Capital (orientador: Prof. Pedro Cezar Dutra Fonseca)

DATHEIN, Ricardo. Experiências de Integração Econômica naAmérica Latina e Cauibe (orientador: Prof. Pedro Cezar Dutra Fonseca).

FARIA, Luiz Augusto Estrella. Um Ensaio Sobre Regulação, Moeda e Inflação no Brasil (orientador: Prof. Pedro Cezar Dutra Fonceca).

FL IGENSPAN, Flávio Benevett. Padrão de Competição e Crescimento da Indústria Gaúcha (1949-1980) (orientador: Prof. Achyles Barcelos da Costa).

FREIRE, Faulo Roberto Rodrigues. Causas da Decadência Econônica da Região de Jaú: um Estudo de Caso (orientador: Prof. Nali de Jesus de Souza).

GARCIAS, Paulo Mello. Os Salários na Induistria de Curtimento de Courose Peles do Rio Grande do Sul: um Estudo de Casos (orientadora: Profa Maria Imilda da Costa e Silva).

GUTIERREZ, Carmen Rosário Ortiz. Os Vendedores Ambulantes na Bolivia: o Comércio do Mercado "La Ramada" (orientador: Prof. Achyles Barcelos da Costa)

HENKIN, Hélio. Inflaçào Alta na América Latina - Uma Análise da Trajetória das Teorias Estruturalistas (orientador: Prof. Roberto Camps Moraes).

HORN, Carlos Henrique Vasconcellos. A Determinação dos Salários e o Pnder de Barganha dos Sindicatos - Mudança Estrutural c Resultadc: das Negociaçōes dos Bancários de Porto Alegre Entre 1979 e 1988 (oricntador: Prof Achyles Barcelos da Costa).

MACADAR, Beky Moron de. A Integração Latino-Americana: da ALALC ao MERCOSUL (orientador: Prof. Achyles Barcelos da Costa).

RUCKERT, Isabel Noema J. O Déficit Público Brasileiro nos Anos 80 - Adequação da Utilização dos Conceitos (orientador: Prof. Joảo Rogério Sanson).

SANTOS, André Maurício dos. Padrão de Crescimento das Empresas do Setor Calçadista do Vale do Sinos (orientador: Prof Ary Burger).

SCHNEIDER, Vera Maria. Os Incentivos Fiscaise as Exportações Gaúchas na Década de 70 (orientador: Prof. Achyles Barcelos da Costa).

SCHETTERT, Maria da Conceição S. e Souza. Cálculo do Indice Trimestral do Produto Real do Rio 
Grande do Sul, 1985-91 (orientador: Prof. Carlos Augusto Crusius).

ZANELLA, Fernando Caputo. Escola Austriaca de Economia Teoria e Método (orientador: Prof. Ernani Hickmann)

ZDANOWICZ, José Eduardo. Perfil e Competitividade da Indístria de Curtumes no Brasil (orientador: Prof. Nali de Jesus de Souza).

STRASSBURGER, Adriano Dirceu. O Impacto das Exportaçáes e do Gasto Público no Desenvolvimento Regional: um Estudo de Caso (orientador: Prof. Nali de de Jesus de Souza).

\section{B) ECONOMIA RURAL}

BEM, Judite Sanson de. Um Estudo Sobre a Comercialização de Hortigranjeiros na Grande Porto Alegre (orientador: Prof. Aray M. Feldens).

BOWLFS, Ricardo Álvaro Gusmán. Avaliação de Alternativas para o Desenvolvimento da Agricultura

Comercial de Santa Cruz de La Sierra-Bolívia (orientador: Prof. Valter José Stulp).

MARTINS, Cid Isidoro de Marco. Ponto de Estrangulamento na Frodutividade da Pecuária Bovina de Corte no Mato Grosso do Sul (orientador: Prof. Atos F. Grawunder).

NETO, Francisco Gelinski. Análise Econômica da Cacaui-cultura Frente a Doença Vassoura de Bruxa - Ihéus, Bahia (orientador: Prof. Reinaldo I. Adams).

RIGATTO, Paulo. Viabilidade Econômica de Sistemas de Produção para Regiōes de Várzeas (orientador: Prof. Valter José Stulp).

RODRIGUES, Luciene. Mecanização Associativa: Alternativa para

Pequenas Propriedades? (O Caso da ADERE em Estrela-RS) (orientador: Prof. Otto G. Konzen).

VELOSO, Gilberto de Oliveira. Determinação de Tamanhos Mínimos de

Propiciedades Rurais Típicas parauma Política de Melhoria de Renda, Emprego e Produção Numa Estratégia de Reforma Agrária no Oeste Catarinense (orientador: Prof. Juvir L. Mattuella).

VIEIRA, Wilson da Cruz. Análise Econômica de Transporte e Armazenagem de Arroz no Estado do Maranhão (orientador: Prof. Aray M. Feldens). 